\title{
A Survey on Recent Techniques for Energy Efficient Routing in WSN
}

\author{
Akila $^{1}$, Uma Maheswari ${ }^{2}$ \\ ${ }^{1}$ Department of Computer Science and Engineering, Mahendra College of Engineering, Salem, India \\ ${ }^{2}$ Department of Computer Science \& Engineering, Anna University, Chennai, India
}

Email address:

akilat.cse@gmail.com (Akila),dr.umasundar@gmail.com (U. Maheswari)

\section{To cite this article:}

Akila, Uma Maheswari. A Survey on Recent Techniques for Energy Efficient Routing in WSN. International Journal of Sensors and Sensor Networks. Vol. 6, No. 1, 2018, pp. 8-15. doi: 10.11648/j.ijssn.20180601.12

Received: November 11, 2017; Accepted: November 22, 2017; Published: January 11, 2018

\begin{abstract}
Wireless Sensor Network is broadly used in many areas including security surveillance. In Wireless Sensor Network, sensor networks are placed randomly and also in grid depending on the methodology used to deploy the network. As sensor nodes uses energy from batteries for sensing the data and transmitting data it consumes the energy for these operations. Avoiding energy consumption in sensor nodes is hot topic in today's era and also a challenging task, many protocols and algorithms are used to avoid energy consumption as the batteries in Wireless Sensor Network are non replaceable. In this paper we made a detailed survey on the recent issues in Wireless Sensor Networks and discussed various problems with respect to different scenarios as well as different methods. Also the paper focuses on recent approaches in avoiding energy consumption of Wireless Sensor Network and researched the different protocols and algorithms by various authors. The paper focuses future study on energy efficiency in Wireless Sensor Network stating new terms and objectives for further research.
\end{abstract}

Keywords: Wireless Sensor Network, Energy Efficiency, Energy Optimization, Sensor Nodes, Routing Protocols

\section{Introduction}

In computer networking, the wireless networks are considered as popular because they are cost effective and installation of these networks are considered to be easy. In the wireless networks, Wireless Sensor Networks (WSN) are popular, in which number of sensor nodes is connected to each other and responsible for communication. The sensor nodes transmit, receive and sense the data as well as form connection with its neighbor nodes or with sink node. [2] These nodes are typically battery-powered, and equipped with low-performance processors and small memories in order to reduce the power requirements. A common sensor node designed using a processing unit, memory chip, power supply and sensors and actuators. The Wireless Sensor Networks are deployed in many places where a man cannot reach easily; most of the application of WSNs is for monitoring forests, underwater 3-D environment, in civil work, also in health and military.

Sensor nodes are powered by batteries and these are non replaceable and non chargeable in this situation energy saving for each sensors nodes is very essential, there are several techniques and protocols used by many researchers so that energy consumption is cut-down to its root and still there are many open challenges in building suitable energy saving techniques for sensor nodes in Wireless Sensor Network's. It is essential to increase the life time of the nodes in Wireless Sensor Network because the use of this network is non reachable for most of the time and replacement of the batteries or defected nodes are impossible. So many researchers have carried out different solutions for energy efficient model in various situations.

The structure of the paper is organized as follows: section 2 discuss more on recent difficulties in energy efficiency as well as stated different problems and occurrences, section 3 is detailed discussion on recent techniques used to improve the energy efficiency in Wireless Sensor Network, section 4 discussed on protocols referred by new techniques for energy saving in Wireless Sensor Network, section 5 concludes about the paper and opens with future topics which helps in building stable energy efficient techniques. 


\section{Related Works}

Wireless Sensor Networks are deployed in different environments and the methodology used to deploy them is different from one to other depending on the applications and needs.

The area of Wireless Sensor Networks research is very vast because the different kind of methods, topologies are used to deploy these, in considering if one protocol is proposed for energy efficient in one environment with respect to the placement of the sensor nodes the same protocol might not work effectively in all other aspects or conditions, some of the techniques proposed by many researchers for a specific network layer and most of the techniques also categorized keeping eye on environment changes.

The solar energy is used to charge the battery of the sensors in some cases which can't be effective in the seasons where sun light is not available constantly. Same as the Wireless Sensor Networks deployed under water for sensing the surrounding cannot work as other designs. So, the different techniques and different algorithms are proposed by various authors with respect to the different scenarios.

The design of Wireless Sensor Networks is different depending on the needs, in some of the Wireless Sensor Networks, the placement of the sensor nodes are completely different. There are different techniques to solve the energy consumption issue for randomly placed sensors and the sensors placed in a grid system.

The techniques further divided based on different network layers, different node densities, co-operative node communication, single input single output as well as multiple inputs and multiple output, fixed distance and variable distance, static WSNs and mobile sink WSNs.

Considering the size of Wireless Sensor Networks when the sensor nodes are placed in smaller regions the effective transmission of data can be achieved easily as the workload is less and more energy can be saved, but when the sensor nodes placed in large regions the transfer rate differs and the energy consumption rate increases as the process of operation increases. Density of the nodes also responsible for energy consumption, also the nodes placed near the sink consumes more energy in large area because every corner sensor nodes transfer the data to the neighbor nodes and the nodes near the sink are too busy in transferring the data which are not free from operating so the energy consumption in this area is more, different techniques of load balancing is used to avoid these kind of issues.

Authors in [3] discussed a well structured research routing protocols based on different scenarios which are

1. Data centric routing protocols

2. Hierarchical routing protocols

3. Location based routing protocols

The issues and the techniques that is separate for separate scenarios because the working of these is totally different from one another

In data centric routing the sink sends the queries to certain regions and waits for data from the sensors located in the selected regions. Since data is being requested through queries, attribute-based naming is necessary to specify the properties of data. Here the data is to be accurate and data redundancy must be avoided in order to save more energy, hence the security for nodes also a challenging area for energy efficient systems [3].

The aim of hierarchical routing is to efficiently maintain the energy consumption of sensor nodes by involving then in multi-hop communication within a particular cluster and by performing data aggregation and fusion in order to decrease the number of transmitted message to the sink [3].

Most of the routing protocols for sensor networks require location information for sensor nodes. In most cases location information is needed in order to calculate the distance between two particular nodes so that energy consumption can be estimated [3].

Considering the different issues in Wireless Sensor Networks there is no single solution for all the issues considering the components used in Wireless Sensor Networks without considering the designs and different methodologies one can achieve best solution on overall issues.

\section{Recent Techniques to Improve the Energy Efficiency}

\subsection{Novel Fuzzy Logic}

Novel based algorithms are good in determining the load balance in WSNs this technique used in [2]. The techniques works well in physical layer and data link layer. It focuses on reducing the whole power consumption while maintaining good performance in terms of the ratio of throughput to workload. This method is used for environment monitoring. Energy consumption is evaluated based on three components i) sensing, ii) processing, iii) transmission. Centralized mechanism is proposed in order to regulate the sleeping time of the field. Activities of microcontroller and of a connected sensor have a low impact on the battery consumption. This approach dynamically changes the sleeping time in order to increase the battery duration of sensor devices. It is not possible to determine the behavior of the nodes since they are often used to monitor sporadic events.

Evaluation of performance in novel fuzzy logic

In both topologies, the best result is obtained by the proposed approach using Gaussian membership functions. In fact, the battery is fully discharged after 169,950 s, i.e. after 47 hours and 21 minutes in the star topology and after is fully discharged after 174, 945s, i.e. after 47 hours and 53 minutes in the cluster topology.

Using the fuzzy-based approach the Th (throughput) / W1 (wavelength) fluctuates between $70 \%$ to $30 \%$ with Gaussian membership functions in the star topology and between $80 \%$ to $45 \%$ in the clustered topology.

Qualities

This improves the lifetime of the devices in WSN and the 
approach dynamically works on changing sleeping time in order to increase the battery life.

What is left?
Need to do simulation to make it suitable also in context characterized by real time constraints.

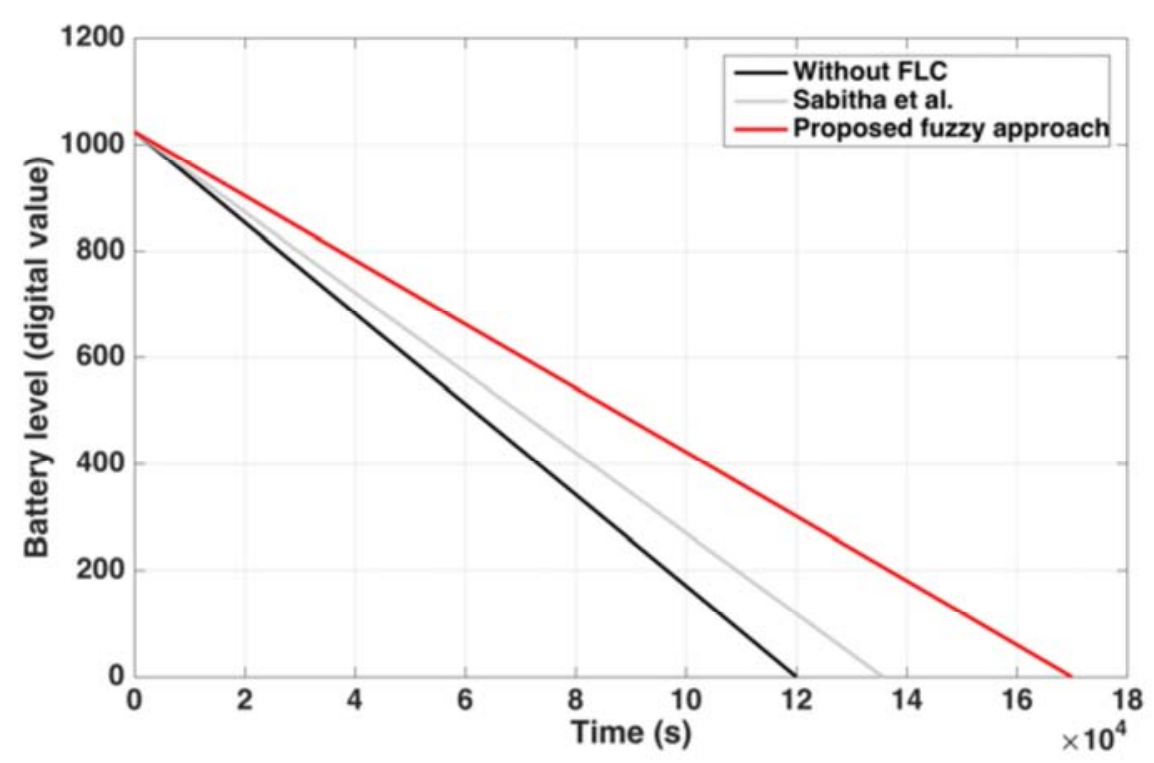

a) Star topology

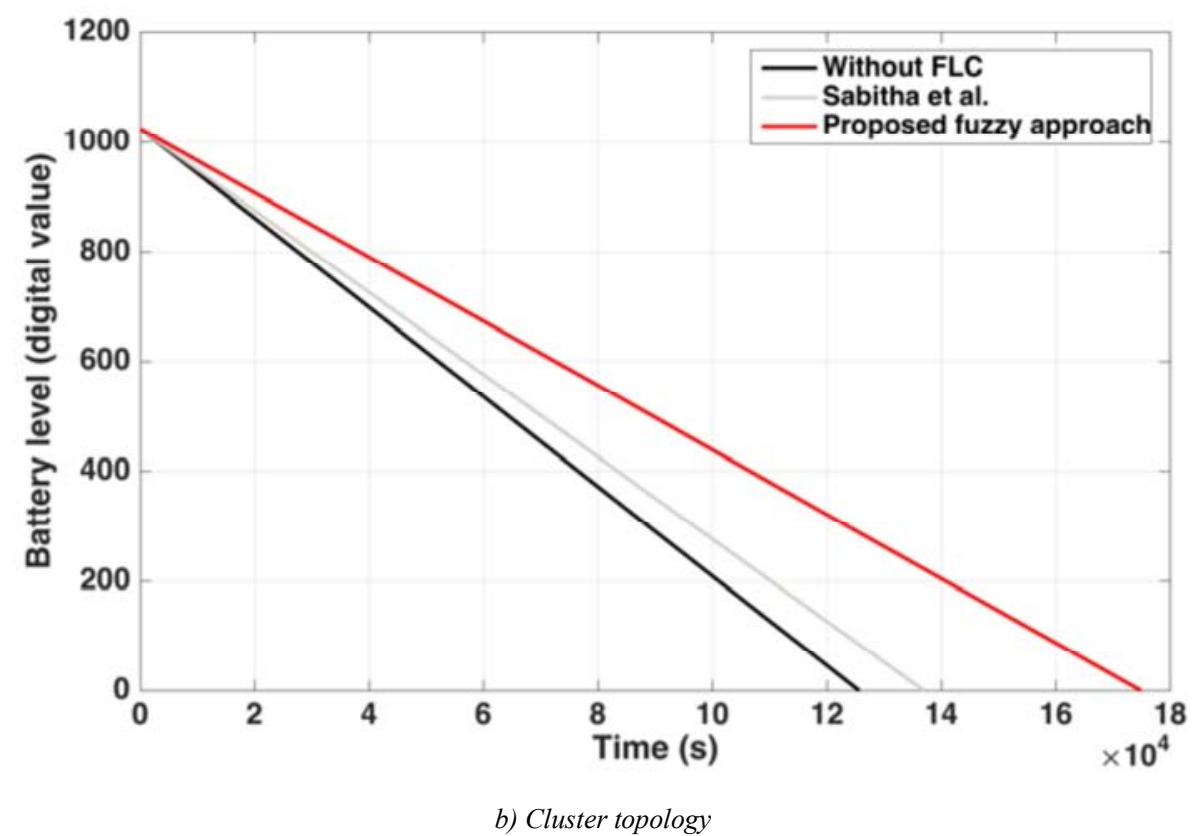

Figure 1. Battery consumption comparison.

\subsection{Delay Aware Routing Protocol}

In [6] author developed the method based on Packet forwarding scheme. The method is used in homogeneous nature. In this sink is GPS enabled. Main activities in this is neighborhood management, deadline estimation, data forwarding Sensor node senses the occurrence of an event or a data packet arrives to it. It is focused on performance comparison between grid and random deployment of sensor nodes. The performance of the protocol can be compared to some of its related protocol.
Performance evaluation of Delay Aware Routing Protocol [6]

It has been observed that the basic behavior of the protocol is almost similar for both the deployment scenarios. However, it is observed some irregularities in case of random deployment while evaluating the performance parameters such as end to end delay, packet delivery ratio and deadline miss ratio. It has been also observed that in some cases random deployment gives better results in comparison to grid deployment.

Performance in Delay routing protocol is mainly based on 
following factors.

1) End to End Delay vs Simulation Time.

2) Packet Delivery Ratio vs Simulation Time.

3) Deadline Miss Ratio vs Number of nodes.

4) Deadline Miss Ratio vs Packet Interval.

5) End to End delay vs Packet Interval.

In the listed scenarios other than last two scenarios other factors only fit for grid deployment and not suitable for random deployment of the nodes.

\subsection{Fixed Competition Based Clustering Approach (FCBA)}

In [7], author proposed a technique based on clustering and $\mathrm{CH}$ (cluster Head) formation, this algorithm includes as follows. It is cluster based. Novel clustering approach used. This protocol is for fixed WSNs. Compared the results with popular approach LEACH which is good in homogeneous distributed clustering. The main issue in any proposed architecture is to maximize the life time of the nodes in WSNs. Selection of the $\mathrm{CH}$ is the key issue in clustering algorithm.

\subsection{Co-operative Multiple Input and Multiple Output- Spatial Modulation (CMIMO-SM)}

In [8], Spatial Modulation is new approach which increases spectral efficiency. Cluster based approach. This method uses only one active antenna during the transmission to avoid Inter Channel Interference (ICI). Combining the CMIMO and SM the WSNs can achieve good energy

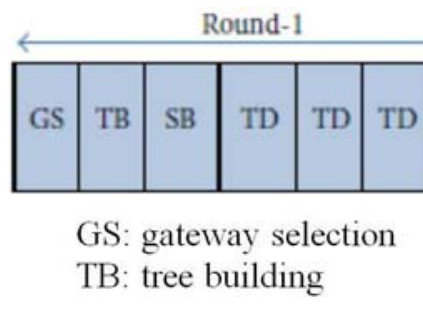

Figure 2. PRRP Phases

\section{Performance Evaluations [9]}

PRRP is similar to LEACH protocol in which any node can communicate with the sink and the data collection or transmission mechanism is the time based schedule. Collectively for all rounds together including 1,5 , and 10 rounds altogether. Simulation results illustrate that with PRRP nodes can stay longer and utilize their maximum possible energy for longer time period. For example, after a 10-round test run, the figure shows that the last node of the LEACH protocol dies after 275 seconds while PRRP nodes start dying after 350 seconds.

\subsection{Energy Aware Routing protocol (PDORP)}

In [10] author, Energy optimization is carried out by using hybrid algorithms. Optimization of communication layers, efficiency. Practical techniques to transmit information are very critical. The main issues in this approach is Bounded only or cluster based architecture.

\subsection{Position Responsive Routing Protocol (PRRP)}

In [9] author used the Cross layer design methodology. In comparing LEACH and CELRP, CHs are selected in novel way. Nodes are distributed in grid, which is used or relevant for forest fire surveillance systems and disaster managements system. Surroundings are tier based cluster location and sink is in middle. Energy consumption is directly proportional to distance. Tree routing mechanism applied (each node transmits the data to its closest node) transmission distance is shortest. A node can only be allowed to join a tree if its energy is sufficient to survive for complete round. Leaf nodes will be "ON" only for one slot just to transfer the data to its parent. Non - leaf will remain "ON" for more slots. This protocol is good for more round of data transmission. Average energy consumed at the initial round is that the three initial phases are for setup and no data transmission during this. This I said as overhead energy. In improving the network lifetime, impressive performance are suggested by author. Nodes are distributed randomly. Each node in this protocol transmits its data to the closest neighbor. Each round of processing time consists of four phases i) gateway selection (GS), ii) Tree building (TB), iii) Schedule building (SB), iv) Transmission of the data. 
Table 1. Performance comparison table of PDORP protocol.

\begin{tabular}{lllllll}
\hline Algorithms Metrics & No. of nodes & DSR & LEACH & PEGASIS & OD-PRRP & PDORP \\
\hline & 100 & 1.739 & 22.858 & 34.792 & 10.789 & 6.878 \\
BER & 200 & 1.766 & 16.363 & 35.329 & 17.112 & 19.932 \\
& 300 & 1.739 & 13.415 & 41.220 & 30.776 & 25.521 \\
& 400 & 1.570 & 23.592 & 36.560 & 26.663 & 20.351 \\
& 500 & 1.668 & 9.029 & 29.590 & 18.825 & 11.595 \\
Delay & 100 & 21.585 & 29.391 & 6.709 & 2.989 & 0.766 \\
& 200 & 17.631 & 29.941 & 8.131 & 6.123 & 5.287 \\
& 300 & 14.415 & 29.185 & 9.915 & 7.234 & 0.538 \\
Energy Consumption & 22.299 & 29.589 & 4.258 & 9.023 & 2.803 \\
& 400 & 10.902 & 29.556 & 1.253 & 9.989 & 0.420 \\
& 500 & 13.454 & 49.921 & 20.518 & 7.996 & 5.969 \\
& 100 & 571.604 & 99.847 & 17.656 & 14.927 & 10.660 \\
Throught & 200 & 23.295 & 149.799 & 13.415 & 5.786 & 0.235 \\
& 400 & 141.511 & 199.699 & 24.492 & 20.892 & 12.488 \\
& 500 & 77.456 & 249.653 & 8.102 & 7.341 & 4.128 \\
& 100 & 28.912 & 38.824 & 0.723 & 4.987 & 0.785 \\
& 200 & 18.175 & 33.190 & 0.342 & 2.221 & 0.509 \\
& 300 & 20.139 & 31.589 & 0.773 & 0.559 & 0.831 \\
\end{tabular}

The method can be applied for the design of several types of sensor networks that require reliability, energy efficiency, scalability, prolonged network lifetime, and low end to end transmission delay without requiring location information e.g. During secured battlefield surveillance, habitat monitoring and underwater monitoring [10].

\subsection{Network Coded Adaptive Duty Cycle}

In [12] author, this system investigates life time improvement approximately $8 \%-14 \%$ and minimizing consumption. Adaptive duty cycle and encoding technique is combined. Works on the area near the sink node, as this is the main bottleneck zone. Simulation results reveal that there is an increase of $2.5 \%$ to $9.5 \%$ of sensor network lifetime by using the proposed adaptive duty cycled network coded wireless sensor network based algorithm, and an increase of $6.5 \%$ to $13.2 \%$ lifetime of wireless sensor network as compared with duty cycled wireless sensor network is achieved. Single hop and multi hop communication used by nodes. $\mathrm{N}$ number of motes distributed uniformly in space. $70 \%$ of motes are adaptive duty cycle enabled. Adaptive cycle is used for switching between active and sleep mode. In the area nodes are divided into two form relay device and network coder motes. Within bottleneck are relay nodes transfer information to the sink. Relay node communicate with other relay node as well as other network coder mote in bottleneck zone using multi-hop communication. The expected issues are works only in the area near the sink node in the WSN.

\subsection{Hybrid Meta Heuristic Based Routing Protocol in WSN}

In [15] author, Generic Algorithm (GA) and Gravitational Search Algorithm (GSA) is used. The algorithms used to determine the next best hop. Objective is to keep sensors operating as long as possible. Generic Algorithm is used to provide efficient clustering by choosing the best node as $\mathrm{CH}$ based on the fitness value. Gravitational Search Algorithm is used to route the data of these CH's to the BS by means of routing by choosing best optimal path. This system is compared with E-OEERP (Enhanced-optimized energy efficient routing protocol) protocol and the results are considered to be good.

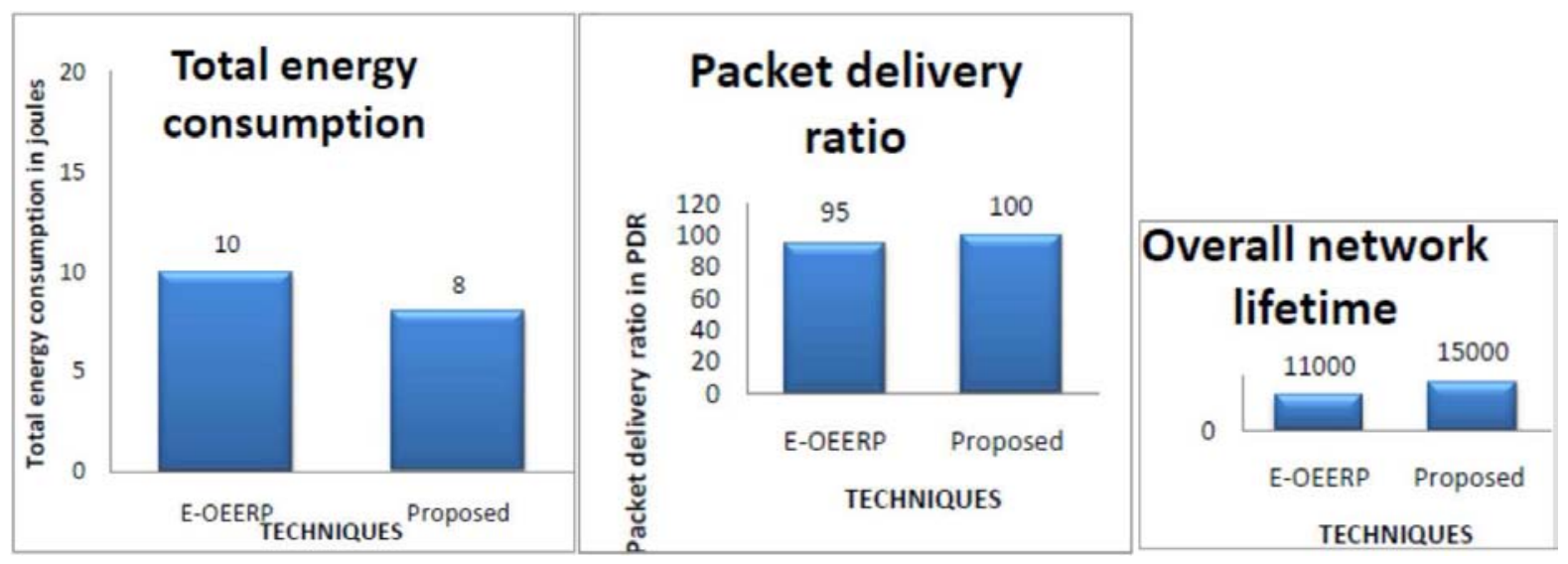

Figure 3. Comparison of various factors with E-OEERP. 


\subsection{Energy Efficient Credit-Based (EECB)}

In [17] author, this Energy Efficient Credit Based protocol selects the most optimal cluster heads based on priority of relay nodes. This protocol has slightly higher packet loss and lower data delivery rate. Such protocol uses candidate nodes for transmission between clusters and uses DirectedDiffusion (DD) for flat routing into hierarchical. DirectedDiffusion (DD) ignores the nodes which are not in the path of sink nodes so, the energy wastage slashed down in this way. Sensors with maximum energy reminders will be selected as Cluster. If the sink node is in the sending limitation of cluster head radius or there is not any relay node, data will be sent to the sink node directly. Otherwise the cluster head selects the node with the highest amount of route choice as the next hop.

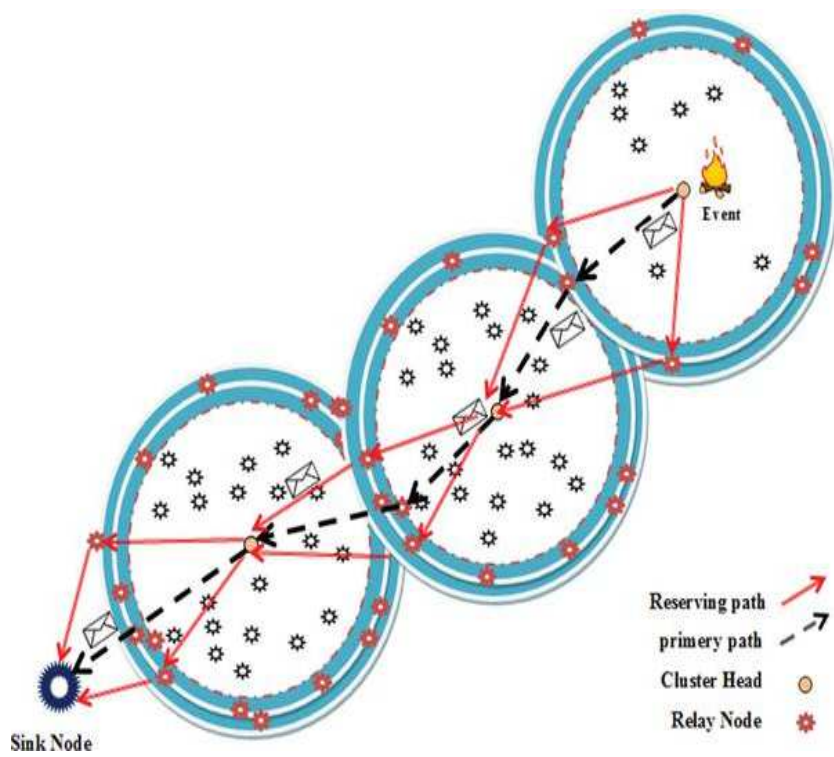

Figure 4. Routing map by clustering and selection of optimum relay node.

The method improved the network lifetime and energy consumption, at the expense of packet loss and data delivery. When the network started, this method achieves a higher data-delivery than LEACH algorithm but after the 100th time slice, LEACH performed better in terms of data delivery and packet loss.

\subsection{Balanced Energy Consuming and Hole Alleviating and Energy Aware BECHA (EA-BECHA)}

In [18] author, this method investigates the energy hole problem where the sensor nodes located near the sink or in some other parts of the network. In other parts of the network nodes die due unbalanced load distribution. This EA-BECHA uses data forwarding and routing selection strategy for the entire network. It assumed that smaller the transmission range more data is forwarded by the sensor nodes located near sink. Energy consumption is mainly dependent on the data size and the distance between them. To maximize the network lifetime it is necessary to maximize the death rate of these sensor nodes. Premature death of sensor nodes will lead to serious energy and coverage hole problem. In Wireless Sensor Networks Energy Hole Alleviating Algorithm (WSNEHA), the energy consumption of the first radius sensor nodes is balanced by the algorithm. However the energy consumption of the sensor nodes located in the other coronas except the first corona is also very uneven. The BECHA is useful for balancing the load distribution between each corona. Performance evaluation for BECHA and EABECHA achieves high packet delivery ratio with less number retransmissions and packet drop ratio due to the low expected error rate.

\section{New Techniques for Energy Saving}

Senor nodes are tiny and typically powered by small batteries for which replacement even if possible is highly expensive and intricate [1]. Every technique proposes includes the common factors like end-to-end delay or end-toend reliability, cluster head formation for larger transmission regions, multipath and single path transmissions, environment based changes, application based protocols.

Equation used in [1] for optimal broadcast bit error probability and hence the optimal long dual distance that maximizes the energy efficient is derived. It is suitable for real time applications, but energy consumption remains constant for traditional fixed distance. Works on node density factors and it is good for lower node density system. [1]

In [3] authors explained list of protocols and categorized then into three parts - i) data centric, ii) Hierarchical, iii) location based. Network flow and QoS also the common factors which are discussed more in every proposed system.

Flooding - without the need of any algorithms and topology sensor nodes broadcast the data.

Gossiping - A nodes sends the packet to a randomly selected neighbor.

LEACH (Low Energy Adaptive Clustering Hierarchy)

A very popular technique to solve the energy consumption issues and the protocol is referred multiple times by various authors. Number of clusters id $5 \%$ of total nodes. It works only on single hop routing and not suitable for large regions.

PEGASIS (Power Efficient Gathering in Sensor Information Systems)

This protocol has out performs compare to LEACH by 100-300\%. Hierarchical PEGASIS is better than PEGASIS improves delayed transmission.

\section{MECN (Minimum Energy Consumption Network)}

Main aim to find sub network which will have less no of nodes and require less power for transmission between any two particular nodes.

\section{GAP (Geographic Adaptive Fidelity)}

Geographic Adaptive Fidelity is energy aware location based routing algorithm for mobile and ad-hoc networks. It turns of unnecessary nodes in the network.

GEAR

This protocol delivers $70-80 \%$ more packets than GPRS in uneven traffic and $25-35 \%$ in the uniform traffic. In [4] 
authors discussed about solar aware routing techniques. In this method the path is static and is not depends on traffic but this protocol is depends on the location of the nodes as the nodes should be in reach of sun light.

MHHC (Multi Hop Hierarchical Clustering)

This protocol improves network life by $22 \%$ than the LEACH. This protocol follows the sequence path according to hierarchical level, and this does not follow the different path to send the information from source to destination. This is basically follows static path. Because of static path the sensor nodes get used repeatedly so the energy level of the sensor nodes will get down and nodes die because of low energy level and hence the network life time also get decreased.

SPIN-I

This protocol approach works as "Blindly forward" and "Data inaccessible" way and this is designed for lossless network based on metadata and not suitable for large network. Transmission time is longer than the SPIN protocol because each node does some calculation before choosing the next hop transmission. Thus helps to balance energy nodes rather than saving it.

Rendezvous Algorithm

This algorithm proposed subset of nodes which server as rendezvous point and aggregates the data from source and transfer to the base station when arrives. This algorithm combines the approach of controlled mobility and data caching in network. In mobility enabled WSN is the increased latency in data collection, there it is giving bottleneck performance.

SPIN (Sensor Protocol for Information Negotiation)

A node advertises for metadata to the destination node. Then the nodes which get the advertisement send the requests back for the data. After that the actual data is transferred to the required node. [16]

TEEN

If the threshold is not reached then the data is not communicated to the base station there were no messages to inform the base station that whether the node has gone dead or the data is not crucial enough to be repeated. APTEEN overcomes this drawback. [16]

Mobile sink nodes over static nodes in WSN

Balancing workload in mobile sink nodes and use of mobile sinks compare to static sinks can greatly improve the energy efficiency of sensor nodes in WSNs. Novel constrained optimization problem faced in this type of techniques. Uses road map connected and all nodes are connected. Mobile sink travel with constant speed on road map. [14]

\section{Conclusion and Future Scope}

\section{Conclusion}

This paper concludes that new techniques and recent studies are improvised in saving the energy and thrashing the bottleneck of energy consumption in WSNs recent studies overall improved by average rate of $25-45 \%$ in saving the energy. Different techniques used in different scenarios based on placing of sensor nodes in WSNs and also based on conditions, application, different environments in every area the respective techniques works well with manageable throughput.

Future Scope

The recent techniques or the researchers focused on protocols by selecting specific area as the WSN is very vast to study. But assuming that finding the solution that should work on all the related structures, topologies, network layers, and different environments is the open topic and using the natural resources in increasing battery life to keep energy efficiency in WSN is also a future step for the study. For under water deployed WSNs finding new techniques in solving the energy consumption issue is also a challenging work.

Overall if we take a close look on future work assumptions there is one technology fits good in all the conditions and that is presumed to be Artificial Intelligent, when protocols using this technique keeping in mind for all the different scenario a robust system can be built.

\section{References}

[1] K. Senthil Kumar and R. Amutha, "An Algorithm for Energy Efficient Cooperative Communication in Wireless Sensor Networks," KSII TRANSACTIONS ON INTERNET AND INFORMATION SYSTEMS, vol. 10, no. 7, July, 2016.

[2] Giovanni Pau, "Power Consumption Reduction for Wireless Sensor Networks Using A Fuzzy Approach," International Journal of Engineering and Technology Innovation, vol. 6, no. 1, pp. 55-67, Dec, 2015.

[3] Kemal Akkaya and Mohamed Younis, "A survey on routing protocols for wireless sensor networks," Computer Science and Electrical Engineering, Ad Hoc Networks 3 (2005) 325349, Nov 2003.

[4] Mr. Santosh N. Shelke, Mr. Sandip R. Shinde, "Energy Saving Techniques in Wireless Sensor Networks," International Journal of Scientific \& Engineering Research, Volume 4, Issue 4, April-2013.

[5] Vasaki Ponnusamy, "Energy Analysis in Wireless Sensor Network: A Comparison," International Journal of Computer Networks and Communications Security, VOL. 2, NO. 9, pp. 328-338, SEPTEMBER 2014.

[6] Bhaskar Bhuyan and Nityananda Sarma, "Performance Comparison of a QoS Aware Routing Protocol for Wireless Sensor Networks," Communications and Network, Feb 2016, $8,45-55$.

[7] Salim EL KHEDIRI, Nejah NASRI and Abdennaceur KACHOURI, "Fixed Competition-based Clustering Approach Wireless Sensor Network," The International Conference on Advanced Wireless, Information, and Communication Technologies (AWICT 2015), Procedia Computer Science 00 (2015) 000-000.

[8] Wided ABIDI and Tahar EZZEDINE, "Optimized CMIMOSM for saving energy in Wireless Sensor Network," IJCSNS, VOL. 16, NO. 10, October 2016. 
[9] Noor Zaman, Low Tang Jung, and Muhammad Mehboob Yasin, "Enhancing Energy Efficiency of Wireless Sensor Network through the Design of Energy Efficient Routing Protocol," Hindawi Publishing Corporation, Volume 2016, Article ID 9278701, May 2016.

[10] Gurbinder Singh Brar, Shalli Rani, Vinay Chopra, Rahul Malhotra, Houbing Song, (Senior Member, IEEE), And Syed Hassan Ahmed, (Student Member, IEEE), "Energy Efficient Direction-Based PDORP Routing Protocol For WSN," Green Communications And Networking For 5g Wireless, July 7, 2016.

[11] Dharini Ganesh, Lalitha M Veeramachaneni and Linda Wong, "Optimization Techniques for Wireless Sensor Networks," George Mason University, INFS 612 - Summer 2009, PGN \# 4.

[12] Shobhit Tyagi and Jitendra Singh Mathur, "Lifetime Improvement of Wireless Sensor Network using Network Coded Adaptive Duty Cycle," IJIRCCE, Vol. 4, Issue 11, November 2016.

[13] K. Hari Krishna, Dr. Y. Suresh Babu and Dr. Tapas Kumar, "Wireless Network Topological Routing In Wireless Sensor Networks," 7th International Conference on Communication, Computing and Virtualization 2016, Procedia Computer Science 79 (2016) 817-826

[14] Jiugen Shi, Xing Wei, and Wei Zhu, "An Efficient Algorithm for Energy Management in Wireless Sensor Networks via Employing Multiple Mobile Sinks," International Journal of Distributed Sensor Networks Volume 2016, Article ID 3179587, December 2015.

[15] Harpreet kaur and Rajeev Vashisht, "Energy Efficient Cluster Based Routing Protocol using Hybrid Metaheuristic in WSNs," International Journal for Science, Management and Technology, ISSN: 2395- 5856, April, 2016.
[16] K. Vinoth Kumar and S. Karthikeyan, "Multihop Energy Efficient Reliable and Fault Tolerant Routing Protocol for Wireless Sensor Networks," International Journal of Emerging Technology and Advanced Engineering, Volume 3, Issue 2, February 2013.

[17] Shahab Tayeb, Miresmaeil Mirnabibaboli and Shahram Latifi, "Cluster Head Energy Optimization in Wireless Sensor Networks," Department of Electrical \& Computer Engineering, University of Nevada, Las Vegas, NV, United States, November 2016.

[18] Nadeem Javaid, "A Balanced Energy Consuming and Hole Alleviating Algorithm for Wireless Sensor Networks," COMSATS Institute of Information Technology, ThesisJanuary 2017.

[19] T. Akila and P. Uma Maheswari, "TQDS: Time Stamped Quantum Digital Signature to Defend Wormhole Attack in Wireless Sensor Network", International Journal of Research in Engineering and Technology, Volume: 03 Special Issue: 07 | May-2014 eISSN: 2319-1163 | pISSN: 2321-7308.

[20] T. Akila and P. Uma Maheswari, "Slashing Energy Consumption Inclusive of Reliable Packet Delivery Via IWDA and DSRA", International Journal of Applied Engineering and Research, ISSN 0973-4562 Volume 10, Number 9 (2015) pp. 21737-21748 (C) Research India Publications.

[21] T. Akila and P. Uma Maheswari, "SEE-AM Route Routing Control Protocol in Green Wireless Sensor Network by GIHBR Algorithm", Asian Journal of Information Technology, Year: 2016 | Volume: 15 | Issue: 3 | Page No.: 437-447 DOI: 10.3923/ajit.2016.437.447. 